\title{
Interactive comment on "RTTOV-gb - Adapting the fast radiative transfer model RTTOV for the assimilation of ground-based microwave radiometer observations" by Francesco De Angelis et al.
}

\section{Anonymous Referee \#1}

Received and published: 27 June 2016

The paper presents an adaptation of the RTTOV radiative transfer model for the ground based observation geometry, called RTTOV-gb. The regression coefficients are computed for a given set of predictors using the same training set that has been used to train RTTOV and the line-by-line absorption model of Rosenkranz 1998. RTTOV-gb has been validated against two radiative transfer models both using line-by-line absorption models and against real observations from a microwave radiometer where RTTOV-gb has been evaluated using colocated radiosonde profiles. The jacobians module of RTTOV-gb has also been validated against ARTS. Finally, RTTOV-gb has been used to perform 1D-var retrievals in a pure simulation environment (OSSE) and the authors

Printer-friendly version

Discussion paper 
could show that the retrievals are closer to the truth than the background.

The paper is very well written, methodologically thorough and scientifically convincing. It is an excellent and very important contribution for the future assimilation of ground based microwave radiometers. It fits very well in the scope of GMD and I recommend to accept the paper for publication subject to some very minor revisions.

Specific comments

P4/I14: not clear if fixed pressure levels are used. I suggest to write "...are pressures of the fixed pressure levels..."

P5/I24: this is better referred to as "linear combination" than "polynomial".

P6/l10: say here which LBL model has been used.

$\mathrm{P} 6 / 110$ : is it justified to say that only 83 profiles cover the variability of humidity and temperature?

P6/I34: use "state vector" instead of "profile vector".

P7//10: y should be bold in the denominator.

P8/15: refer to table 2.

P8/I31: in this and the following sentence you use twice "underestimate" which does not seem to make sense. Please check.

P10/18: why does it make sense to compare RTTOV-gb to two other implementations of R98? Would it not be more sensible to use different absorption models. For ARTS one could use individual LBL calculation rather than the predefined models. Please comment.

Printer-friendly version

Interactive comment on Geosci. Model Dev. Discuss., doi:10.5194/gmd-2016-65, 2016.

Discussion paper 